\title{
Prognostic Value of Subcarinal Lymph Nodes in Minimally Invasive Esophagectomy for Cancer
}

\author{
ELIAS K. SDRALIS ${ }^{1,2}$, SPYRIDON DAVAKIS ${ }^{1,3}$, ATHANASIOS SYLLAIOS ${ }^{1,3}$, \\ BRUNO LORENZI ${ }^{1}$, ALI KORDZADEH ${ }^{1}$, ANTONIOS ATHANASIOU ${ }^{3}$, STAMATIOS PETOUSIS $^{2}$, \\ THEODOROS LIAKAKOS ${ }^{3}$ and ALEXANDROS CHARALABOPOULOS ${ }^{1,3}$ \\ ${ }^{1}$ Regional Esophahagogastric Centre, Department of Upper Gastrointestinal Surgery, \\ Broomfield Hospital, Mid Essex Hospital Services NHS Trust, Chelmsford, U.K.; \\ ${ }^{2}$ Department of Surgery, 424 General Military Hospital, Thessaloniki, Greece; \\ ${ }^{3}$ First Department of Surgery, Laiko General Hospital, \\ National and Kapodistrian University of Athens, Athens, Greece
}

\begin{abstract}
Background/Aim: Surgical resection for esophageal cancer has remained the gold-standard therapy provided worldwide. Subcarinal lymph-nodes are classified as periesophageal nodes and their dissection may be performed during a 2- or 3-stage esophagectomy. The necessity and prognostic value of subcarinal lymphadenectomy is still debatable. The purpose of this study was to investigate the rate of invaded subcarinal lymph-nodes out of the total lymphnodes resected. Detection of invaded nodes was correlated with clinical staging and tumor differentiation. Patients and Methods: This is a retrospective analysis of consecutive esophagectomies for cancer, performed in a UK tertiary center. The study was conducted over a 3-year period. Results: The rate of subcarinal lymph-node invasion was extremely low according to the results of our analysis. Conclusion: Lymphadenectomy is not devoid of clinical impact on patients and therefore, the decision should actually be the outcome of a dynamic balance between complications and survival benefit.
\end{abstract}

Esophageal cancer remains one of the most commonly diagnosed malignancies worldwide, characterized by increased rates of morbidity and mortality (1). The estimated number of 456,000 new cases and 400,000 deaths in 2012 is characteristic of the increasing incidence and significance of the disease (2). Esophageal cancer mortality may not only be attributed to its

Correspondence to: Spyridon Davakis MD, General Surgery Registrar, First Department of Surgery, Laiko General Hospital, National and Kapodistrian University of Athens, 17 Agiou Thoma Str., 11527 Athens, Greece. Tel: +30 6973762451, e-mail: spdavakis@gmail.com

Key Words: Subcarinal, lympadenectomy, esophageal cancer, minimally-invasive. biological behavior, but also to the fact that it is commonly diagnosed at an advanced stage, therefore necessitating radical treatment and deteriorating prognosis $(1,3,4)$.

Despite the fact that various approaches have been proposed for the treatment of esophageal cancer, surgical resection has remained the gold-standard therapy provided (5). Apart from radical esophagectomy, standard lymph-node dissection should accompany surgical approach, as nodal status has been described as a main independent predictor of survival. Surgical approach should be based on the distribution pattern of lymphnode metastases, which remains unpredictable; there is no worldwide consensus on the extent of lymphadenectomy (6).

Lymphadencetomy in esophagectomy has previously been described as standard 2-field, extended 2-field, total mediastinal and 3-field; subcarinal lymph-nodes are classified as peri-esophageal nodes. As a result, dissection of the subcarinal lymph-nodes may be performed during 2- or 3stage esophagectomy, but it is not performed during transhiatal esophagectomy.

Some authors attribute extensive lymphadenectomy to the increased morbidity associated with esophagectomy, which has recently been shown to have implications in the longterm oncological outcome. On these grounds, the necessity and prognostic as well as therapeutic value of subcarinal lymphadenectomy is still debatable (7-9).

The main objective of this study was to assess the metastatic subcarinal lymph-node rate and therefore discuss the necessity of their dissection during the standard 2- or 3field lymphadenectomy for esophageal cancer.

\section{Patients and Methods}

This is a retrospective analysis of prospectively collected data, regarding $n=66$ consecutive esophagectomies for cancer, performed in a UK tertiary referral center. The study was conducted over a 3- 
year period between January 1st 2014 and December 31st 2016. All procedures followed were in accordance with the ethical standards of the Helsinki Declaration of 1964 and later versions. Informed consent or substitute for it was obtained from all patients for being included in the study.

Included patients were aged 18 and above and were diagnosed with invasive cancer (adenocarcinoma or squamous cell carcinoma) or high-grade dysplasia of the mid or distal esophagus/esophagogastric junction (Siewert I-II). Cases with recurrent disease, cancer of the cervical esophagus as well as all esophagectomies during the study period that were performed for benign disease were excluded from the study.

All patients were staged with positron emission tomographycomputed tomography (PET-CT), endoscopic ultrasound (EUS), computed tomography (CT). Furthermore, staging laparoscopy was performed for the adenocarcinoma cases. All patients were preoperatively assessed with Cardio-pulmonary Exercise Test (CPET) to gauge surgical fitness for resectability.

Patients were offered esophagectomy with standard lymph-node dissection from the same surgical team. Procedures performed were: Hybrid 2-stage-esophagectomy (laparoscopic/thoracotomic) with 2field lymph-node dissection, totally minimally-Invasive 2-stageesophagectomy (laparoscopic/thoracoscopic) with 2-field lymphnode dissection, Hybrid 3-stage Minimally-Invasive esophagectomy (laparoscopic/thoracotomic/left neck) with 3-field lymph-node dissection and totally minimally-invasive 3-stage-esophagectomy (laparoscopic/thoracoscopic/left neck) with 3-field lymph-node dissection.

The purpose of the present study was to investigate the rate of invaded subcarinal lymph-nodes out of the total lymph-nodes resected. Detection of metastatic subcarinal lymph-nodes was correlated with clinical staging and tumor differentiation. Surgical records were reviewed and epidemiological characteristics regarding age, gender and ethnicity were retrieved. Furthermore, surgical approach, preoperative and final histopathological staging along with tumor differentiation were further analyzed.

Clinical data were collected through medical records and by personal interview during follow-up.

Descriptive statistical analysis was conducted for all of the encountered parameters, measuring the accumulated values; all repeated measures are expressed as Mean values \pm Standard Deviation, with reference to its corresponding range. All results were analyzed using the SPSS v22.0 (Statistical Package for the Social Sciences Inc., Chicago, IL, USA).

\section{Results}

Within the study period, $n=66$ consecutive patients were treated with esophagectomy for cancer or high-grade dysplasia of the esophagus. Mean patient age was $64.6 \pm 9.8$ years. Majority were males $(90.9 \%, \mathrm{n}=60)$.

Preoperative biopsies revealed adenocarcinoma in $\mathrm{n}=62(93.9 \%)$ and squamous-cell carcinoma in $\mathrm{n}=1 . \mathrm{N}=3$ (4.5\%) had high-grade dysplasia (HGD), $\mathrm{n}=47$ (71.2\%) were poorly differentiated, $\mathrm{n}=16(24.2 \%)$ were moderately differentiated and $n=3(4.5 \%)$ had well differentiated tumors. Tumour location was the mid-esophagus in $n=4$, distal esophagus in $n=22$ and esophagogastric-junction (Siewert III) in the remaining $n=40$.
PET-CT scanning was positive for lymph-node metastasis in $n=29$ patients $(43.9 \%)$. Vast majority of patients $(n=28$, $42.4 \%$ ) were pre-operatively staged as T3N1 by EUS and PET-CT collectively. Staging laparoscopy did not reveal peritoneal disease or distant metastases in any patient.

$\mathrm{N}=45$ (68.2\%) patients received neoadjuvant chemotherapy with 3-cycles of EOX (epirubicin/oxaliplatin/ capecitabine).

$\mathrm{N}=36(54.5 \%)$ underwent Hybrid 2-stage esophagectomy, $\mathrm{n}=26(39.4 \%)$ totally minimally-invasive 2-stage esophagectomy, $\mathrm{n}=2(4.5 \%)$ hybrid minimally-invasive 3-stage esophagectomy and the remaining $n=2$ (4.5\%) underwent totally minimally-invasive 3 -stage esophagectomy.

Patients' demographics, pre-operative staging and histopathological characteristics, are provided in Table I.

Out of the 1.808 lymph-nodes resected, $n=173(9.5 \%)$ subcarinal lymph-nodes were harvested. The median number of resected nodes was $n=23$ (range $=8-65$ ), with median subcarinal nodes being $\mathrm{n}=2$ (range $=0-13$ ).

The overall rate of subcarinal lymph-node invasion was $0.57 \%$, with only $\mathrm{n}=1$ being metastatic, making thus the median positive subcarinal lymph-node number null. On the other hand, overall rate of any lymph-node invasion was $12.8 \%$. Median number of invaded lymph-nodes was $n=3$ (range=0-36). Lymph-node status (histopathological) is mentioned in Table II. The sole patient with an invaded subcarinal lymph-node harvested, had a T3N3 squamous cell carcinoma of the mid-esophagus and had previously undergone neo-adjuvant chemotherapy.

Univariate analysis indicated that lymph-node invasion as well as total number of resected nodes were significantly correlated with postoperative TNM staging $(p=0.03)$ and tumor differentiation $(p=0.04)$. No significant correlation $(p<0.05)$ between lymph-node invasion and age, gender or any other epidemiological parameter was observed. Univariate regression analysis is shown in Table III.

\section{Discussion}

Up to date, esophagectomy remains the gold-standard treatment for esophageal cancer, however the extend of lymph-node dissection has not yet been standardized. Lymphatic drainage of the oesophagus runs in the longitudinal direction, resulting in regional, cephalad/caudal bidirectional metastases (10). Lack of serosa in the esophagus allows local radial extension of the tumor. Regional lymphatic drainage has not yet been entirely elucidated; worldwide there is no uniform consensus on the definition of regional lymph-node resection. Subcarinal lymph-nodes are considered regional in esophageal cancer (11); thus, during esophagectomy, their dissection has become a routine step. However, few studies have reported the rate of subcarinal lymph-node metastasis in intra-thoracic or intra-abdominal esophageal carcinoma. 
Table I. Epidemiological and clinical characteristics of the study population.

\begin{tabular}{lc}
\hline Characteristics & Patients $(\mathrm{n}=66)$ \\
\hline Age (Mean \pm SD) & $64.6 \pm 9.8$ years \\
Gender & \\
Male & $\mathrm{n}=60(90.9 \%)$ \\
Female & $\mathrm{n}=6(8.1 \%)$ \\
Management & \\
Hybrid 2-stage-esophagectomy & $\mathrm{n}=36(54.5 \%)$ \\
Totally minimally-invasive 2-stage-esophagectomy & $\mathrm{n}=26(39.4 \%)$ \\
Hybrid minimally-invasive 3-stage-esophagectomy & $\mathrm{n}=2(4.5 \%)$ \\
Totally minimally-invasive 3-stage-esophagectomy & $\mathrm{n}=2(4.5 \%)$ \\
Neoadjuvant chemotherapy & $\mathrm{n}=45(68.5 \%)$ \\
PET-CT scanning positive lymph-nodes & $\mathrm{n}=29(43.9 \%)$ \\
Histological type & \\
Adenocarcinoma & $\mathrm{n}=62(93.9 \%)$ \\
Squamous cell carcinoma & $\mathrm{n}=1(1.5 \%)$ \\
High-grade dysplasia & $\mathrm{n}=3(4.6 \%)$ \\
Differentiation & \\
Poor & $\mathrm{n}=47(71.2 \%)$ \\
Moderate & $\mathrm{n}=16(24.2 \%)$ \\
Well-differentiated & $\mathrm{n}=3(4.6 \%)$ \\
Preoperative TNM-staging & \\
T1N0M0 & $\mathrm{n}=4(6.1 \%)$ \\
T2N0M0 & $\mathrm{n}=10(15.2 \%)$ \\
T3N0M0 & $\mathrm{n}=9(13.6 \%)$ \\
T2N1M0 & $\mathrm{n}=11(16.7 \%)$ \\
T3N1M0 & $\mathrm{n}=7(1.5 \%)$ \\
T3N2M0 & $\mathrm{n}=1(1.5 \%)$ \\
T3N3M0 & $\mathrm{n}=28(42.4 \%)$ \\
Non-specified & $\mathrm{n}=0)$ \\
Postoperative TNM staging & $\mathrm{n}=1(1.5 \%)$ \\
T0N0M0 & $\mathrm{n}=11(16.7 \%)$ \\
T1N0M0 & $\mathrm{n}=7(10.6 \%)$ \\
T1N1M0 & $\mathrm{n}=7(10.6 \%)$ \\
T1N2M0 & $\mathrm{n}=2(3.0 \%)$ \\
T2N0M0 & \\
T3N0M0 & \\
T2N1M0 & \\
T3N1M0 & \\
T3N2M0 & \\
T3N3M0 & \\
T4N3M0 & \\
\hline &
\end{tabular}

The majority of surgeons perform an extended mediastinal lymphadenectomy including the subcarinal area. However, others report limited lymphadenectomy preserving the subcarinal lymph-nodes in patients that have undergone neoadjuvant chemotherapy. Recently, in Europe, apart from the two-stage (Ivor-Lewis) or 3-stage (McKeown) esophagectomy, it has been reported that up to $40 \%$ of patients still undergo transhiatal resection, during which the mediastinal lymphnodes are not dissected (6).

It has been postulated that extensive lymph-node dissection increases surgical trauma and incidence of post-
Table II. Primary and secondary study outcomes.

\begin{tabular}{lc}
\hline Characteristics & Number $(\%)$ \\
\hline LN resected & $\mathrm{n}=1808$ \\
Subcarinal LN resected & $\mathrm{n}=173(9.5 \%)$ \\
Median LN resected & $\mathrm{n}=23($ range $=8-65)$ \\
Median subcarinal LN resected & $\mathrm{n}=2$ (range $=0-13)$ \\
Number of metastatic LN's & $\mathrm{n}=231(12.8 \%)$ \\
Number of metastatic subcarinal LN's & $\mathrm{n}=1(0.57 \%)$ \\
Median metastatic LN's resected & $\mathrm{n}=3($ range $=0-36)$ \\
\hline
\end{tabular}

LN: Lymph nodes.

Table III. Univariate regression analysis between epidemiologicalhistological parameters and lymph node invasion.

\begin{tabular}{lc}
\hline Characteristics & $p$-Value \\
\hline Age & 0.59 \\
Gender & 0.62 \\
Preoperative chemotherapy & 0.25 \\
Tumor differentiation & 0.04 \\
Postoperative stage & 0.03 \\
\hline
\end{tabular}

operative complications (12). Additionally, subcarinal dissection significantly increases operating time, blood loss and postoperative pleural drainage volume (13).

Great controversy exists in the published literature, regarding the issue of subcarinal lymph-node involvement. In our analysis, the rate of subcarinal node invasion was extremely low in a series of 66 consecutive esophagectomies for cancer or high-grade dysplasia. Niwa et al., in a retrospective analysis of 342 patients undergoing R0 subtotal esophagectomy estimated the rate to be $7.0 \%$ (14). Similarly, Liu et al., in a retrospective analysis of 1,800 patients demonstrated an overall invasion rate of approximately $10.0 \%$ (15). On the other hand, Feng et al., in a retrospective series of 507 patients observed an invasion rate of $22 \%$ (16). However, the latter retrospective series of patients, included only squamous cell carcinomas, which may explain the relatively increased rate of subcarinal node invasion. The results of all the aforementioned studies are not consistent with ours, showing an invasion rate close to zero.

As there are no large prospective or randomized controlled studies, the issue is still of high clinical and scientific importance, while the actual rate of subcarinal invasion may be related to the primary location of the carcinoma, its histological type, the tumor differentiation and TNM staging.

Literature is also inconclusive regarding the potential prognostic value of subcarinal nodes. In $\mathrm{N}$ positive disease, 
$\mathrm{Hu}$ et al. found no statistically significant difference in overall survival between patients that had undergone subcarinal lymph-node dissection and those without (13). They reported that subcarinal dissection could increase the survival rate of patients with intra-thoracic esophageal squamous cell carcinomas. Niwa et al., in a retrospective study, indicated that the efficacy index (EI) for the upper and lower thoracic esophagus was 0.0 (14). Furthermore, they demonstrated that 5-year disease-free survival was significantly lower in patients with pathological subcarinal lymph node metastasis than in those without. Moreover, when adjusting for covariates in a multivariate analysis, only clinical $\mathrm{T}$ stage (T2-T4) was the independent predictive factor for pathological subcarinal lymph-node metastases. Therefore, subcarinal lymph-node dissection may actually be of limited significance especially for superficial carcinomas.

Results of Feng et al. and Liu et al. $(15,16)$ rather stand in favor of subcarinal lymph-node dissection. Indeed, Feng et al. demonstrated that subcarinal lymph-node metastases were significantly associated with increased 5-year survival rates, as there was almost a 3-fold decrease in survival of patients with invaded subcarinal lymph-nodes. A relative conclusion was also made by Liu et al. Authors observed that patients with solitary subcarinal node metastasis had a significantly lower 5-year cumulative survival rate than those with solitary paraesophageal node metastasis. However, rates were $25.3 \%$ vs. $39.6 \%$, respectively, while the respective percentages in the study by Feng et al. were $26.7 \%$ vs. $60.9 \%$ (14-16). A potential explanation regarding the differences in their results may actually lie in the fact that the study by Feng et al. included squamous cell carcinomas, which are by definition of much worse prognosis in comparison with other histological types (17-20).

Our study has several limitations including being a retrospective one. There are still no definitive data on the 5year survival rate, as the first patients were operated in 2014 and the last ones in 2016. Furthermore, the majority of cases were adenocarcinomas, which have a rather lower-risk of lymph-node metastases in comparison with other histological sub-types. The main advantage of this study is its uniformity regarding the operating steps and the fact that all cases underwent surgery in a very similar fashion by one operating team following standardized oncological principles. There is a minute number of studies investigating the actual rate of subcarinal lymph-node involvement in esophagectomy for cancer.

\section{Conclusion}

In summary, the rate of subcarinal lymph-node invasion is extremely low according to the results of our analysis. The prognostic role may be more important than the therapeutic one for lymphadenectomy in esophageal cancer surgery. In other words, patients with lymph node metastasis are suffering from systemic disease and the attempt to cure them by also performing lymphadenectomy may be unrealistic. Further prospective high-power studies are needed to clarify this significant issue. Lymphadenectomy is not devoid of clinical impact on patients and therefore, the decision should actually be the final outcome of a dynamic balance between complications and benefit in survival.

\section{Conflicts of Interest}

The Authors report no conflict of interest regarding this study.

\section{Authors' Contributions}

Elias K. Sdralis: drafting of manuscript and analysis and interpretation of data; Spyridon Davakis: drafting of manuscript and analysis and interpretation of data, revision of manuscript; Athanasios Syllaios: drafting of manuscript; Bruno Lorenzi: acquisition of data; Ali Kordzadeh: analysis and interpretation of data; Antonios Athanasiou: acquisition and data analysis; Stamatios Petousis: drafting of manuscript; Theodoros Liakakos: study conception and data acquisition; Alexandros Charalabopoulos: study conception and design, acquisition of data, drafting of manuscript and critical revision of manuscript.

\section{References}

1 Domper Arnal MJ, Ferrández Arenas Á and Lanas Arbeloa Á: Esophageal cancer: Risk factors, screening and endoscopic treatment in Western and Eastern countries. World J Gastroenterol 21(26): 7933-7943, 2015. PMID: 26185366. DOI: 10.3748/wjg.v21 i26.7933

2 Arnold M, Laversanne M, Brown LM, Devesa SS and Bray F: Predicting the future burden of esophageal cancer by histological subtype: International trends in incidence up to 2030. Am J Gastroenterol 112(8): 1247-1255, 2017. PMID: 28585555. DOI: 10.1038/ajg.2017.155

3 Zhang HZ, Jin GF and Shen HB: Epidemiologic differences in esophageal cancer between Asian and Western populations. Chin J Cancer 31(6): 281-286, 2012. PMID: 22507220. DOI: $10.5732 /$ cjc. 011.10390

4 Wheeler JB and Reed CE: Epidemiology of esophageal cancer. Surg Clin North Am 92: 1077-1087, 2012. PMID: 23026270. DOI: $10.1016 /$ j.suc.2012.07.008

5 Kato $\mathrm{H}$ and Nakajima M: Treatments for esophageal cancer: a review. Gen Thorac Cardiovasc Surg 61: 330-335, 2013. PMID: 23568356. DOI: $10.1007 / \mathrm{s} 11748-013-0246-0$

6 Hagens ERC, van Berge Henegouwen MI, Cuesta MA and Gisbertz SS: The extent of lymphadenectomy in esophageal resection for cancer should be standardized. J Thorac Dis 9: S713-S723, 2017. PMID: 28815067. DOI:10.21037/jtd.2017. 07.42

7 Low DE: Evolution in surgical management of esophageal cancer. Dig Dis 31: 21-29, 2013. PMID: 23797119. DOI: $10.1159 / 000343650$

8 Vallbohmer D, Oh DS and Peters JH: The role of lymphadenectomy in the surgical treatment of esophageal and gastric 
cancer. Curr Probl Surg 49: 471-515, 2012. PMID: 22793506. DOI: $10.1067 /$ j.cpsurg.2012.04.003

9 Borggreve AS, Kingma BF, Domrachev SA, Koshkin MA, Ruurda JP, van Hillegersberg R, Takeda FR and Goense L: Surgical treatment of esophageal cancer in the era of multimodality management. Ann NY Acad Sci 1434(1): 192209, 2018. PMID: 29761863. DOI: 10.1111/nyas.13677

10 Sharma D, Thakur A, Toppo S and Chandrakar SK: Lymph node counts in indians in relation to lymphadenectomy for carcinoma of the oesophagus and stomach. Asian J Surg 28(2): 116-120, 2005. PMID: 15851365. DOI: 10.1016/S1015-9584(09)60274-8

11 Tachibana M, Kinugasa S, Yoshimura H, Dhar DK and Nagasue $\mathrm{N}$ : Extended esophagectomy with 3-field lymph node dissection for esophageal cancer. Arch Surg 138(12): 1383-1389, 2003. PMID: 14662544. DOI: 10.1001/archsurg.138.12.1383

12. Law $\mathrm{S}$ and Wong J: Lymph node dissection in surgical treatment of esophageal neoplasms. Surg Oncol Clin N Am 16: 115-131, 2007. PMID: 17336240. DOI: 10.1016/j.soc.2006.10.008

13. Hu W, Liang Y, Zhang S, Hu Y and Liu J: The significance of subcarinal dissection in esophageal cancer surgery. Asia Pac J Clin Oncol 10(2): 183-189, 2014. PMID: 23915120. DOI: 10.1111/ajco.12095

14. Niwa Y, Koike M, Hattori M, Iwata N, Takami H, Hayashi M, Tanaka C, Kobayashi D, Kanda M, Yamada S, Fujii T, Nakayama G, Sugimoto H, Fujiwara M and Kodera Y: The prognostic relevance of subcarinal lymph node dissection in esophageal squamous cell carcinoma. Ann Surg Oncol 23: 611618, 2016. PMID: 26289807. DOI: 10.1245/s 10434-015-4819-2

15 Feng JF, Zhao Q and Chen QX: Prognostic value of subcarinal lymph node metastasis in patients with esophageal squamous cell carcinoma. Asian Pac J Cancer Prev 14: 3183-3186, 2013. PMID: 23803101.
16 Liu J, Hu Y, Xie X and Fu J: Subcarinal node metastasis in thoracic esophageal squamous cell carcinoma. Ann Thorac Surg 93: 423-427. PMID: 22178587. DOI: 10.1016/j.athoracsur. 2011.10.011

17 Chen SB, Weng HR, Wang G, Yang JS, Yang WP, Li H, Liu DT and Chen YP: Basaloid squamous cell carcinoma of the esophagus. J Cancer Res Clin Oncol 138: 1165-1171, 2012. PMID: 22419439. DOI: 10.1007/s00432-012-1180-8

18 Chen SB, Weng HR, Wang G, Yang JS, Yang WP, Liu DT, Chen YP and Zhang H: Primary adenosquamous carcinoma of the esophagus. World J Gastroenterol 19: 8382-8390. PMID: 24363531. DOI: $10.3748 /$ wjg.v19.i45.8382

19 Zhang HD, Chen CG, Gao YY, Ma Z, Tang P, Duan XF, Ren P, Yue $\mathrm{J}$ and Yu ZT: Primary esophageal adenosquamous carcinoma: a retrospective analysis of 24 cases. Dis Esophagus 27: 783-789, 2014. PMID: 24127755. DOI: 10.1111/dote.12153.

20 Sun $\mathrm{YH}$, Lin SW, Chen $\mathrm{CH}$, Liang WY and Hsieh CC: Adenosquamous carcinoma of the esophagus and esophagogastric junction: clinical manifestations and treatment outcomes. J Gastrointest Surg 19(7): 1216-1222, 2015. PMID: 25967141. DOI: $10.1007 / \mathrm{s} 11605-015-2852-\mathrm{x}$
Received May 24, 2019

Revised June 12, 2019

Accepted June 13, 2019 